\title{
Congress Award Lecture
}

\section{Development and Analysis of Disease Models Using Gene Targeting Techniques.}

\author{
Yoichiro Iwakura \\ Center for Experimental Medicine, Institute of Medical Science, University of Tokyo,Research Institute for Biological Sciences, Tokyo \\ University of Science
}

I succeeded in generating transgenic (Tg) mice carrying the interferon beta gene for the first time in the world, and published in EMBO J. in 1988. Then, I generated Tg mice carrying the tax gene of HTLV-I, which is the causative agent of adult T cell leukemia. Because these transgenic mice spontaneously developed autoimmune arthritis resembling rheumatoid arthritis in humans, I suggested that HTLV-I is one of etiological agents of rheumatoid arthritis (Science, 1991). Through the analysis of these Tg mice, I found that Tax-induced IL-1 might play a critical role in the pathogenesis of arthritis. Then, we knocked out all the IL-1 molecules, i.e. IL-1 $\alpha$, IL-1 $\beta$, IL- $1 \alpha / \beta$, and IL-1 receptor antagonist (IL-1Ra). We found that IL-1 $\beta$ plays a critical role in the febrile response and glucocorticoid induction under stress (J. Exp. Med., 1988). Furthermore, we found that IL-1Radeficient (KO) mice develop arthritis and aortitis, suggesting excess IL-1 signaling induces autoimmunity (J. Exp. Med., 2000; Circulation, 2005). Because both arthritis and aortitis were not developed in TNF-deficient-IL-1Ra KO mice, we suggested that TNF is a good candidate for the treatment of these diseases (J. Clin. Invest., 2004). We also generated IL-17-deficient mice, because this gene expression was enhanced in both HTLV-I Tg and IL-1Ra KO mice. We showed that the development of arthritis was strongly suppressed in IL-17 KO mice, suggesting that IL-17 plays a critical role in the development of arthritis (Immunity, 2002; PNAS, 2003). Furthermore, we showed that IL-17F, the molecule with the highest homology with IL-17A in the same family, is important for the host defense against microbial mucoepithelial infection (Immunity, 2009; Immunity, 2011). To further analyze the mechanisms of the development of autoimmunity and arthritis, we knocked out genes which were highly expressed in arthritic joints. Among them, we showed that Dectin-1 and Dectin-2, C-type lectin receptors, is the receptor for $\beta$-glucans and $\alpha$-mannan, respectively, and play important roles in the host defense against fungal infection (Nat. Immunol., 2007). Interestingly, Th17 cell differentiation was preferentially induced by these signals, suggesting that fungal infection may cause autoimmunity (Immunity, 2010). We also showed that KO mice of Dcir, another C-type lectin family member, develop autoimmune enthesitis and sialadenitis (Nat. Med., 2008). These results indicate that non-TLR innate immune molecules such as C-type lectins are important for the regulation of the acquired immune system and can be a good target for the treatment of autoimmune diseases. Taken together, I could show through these studies that gene manipulated mice are useful for the development of disease models and analysis of the pathogenic mechanisms. 
Exp. Anim. 61(3), 203, 2012

\section{Congress Award Lecture}

\section{Establishment of an Efficient-banking System of Genetically Engineered Mouse Using Sperm Cryopreservation}

Toru Takeo

Center for Animal Resources and Development, Kumamoto University

Since finding the double helix of DNA, the analysis of gene function has been big and significant challenge for mankind to advance in life science. With emerging genetically engineered mice, various functions of gene in mammalian has been revealed and provided us with useful information to elucidate physiological phenomenon and find disease-responsible gene and potential drug target. Now, international projects of generating knockout mouse in every gene of mouse genome are going on.

Mouse bank which is the center of mouse science and the hub facility of mouse logistics has established worldwide. One of the most important roles of mouse bank is a large-scale archiving system of mouse with reproductive technology. Cryopreservation of embryos and sperm are used for archiving the mice, which can save the space and cost for breeding mice and comply with the animal welfare act. Especially, sperm cryopreservation is preferable to preserve the samples. So it can be easily performed all procedures, preserved many germplasm from a male and produce over 1,000 progeny via in vitro fertilization (IVF) and embryo transfer.

Success of sperm cryopreservation in mouse was firstly reported at 1990. Subsequently, Nakagata et al. developed a reliable method of sperm cryopreservation, so called Nakagata's method, which has been used for standard method in mouse banks worldwide $^{1}$. In sperm cryopreservation, it is well known that the fertility of frozen-thawed sperm is strain-dependent. Most of inbred and F1 hybrid strains of sperm indicate normal fertility after freezing and thawing. However, the fertility of frozen-thawed sperm in C57BL/6 mouse which is the major inbred strain used for producing genetically engineered mouse is quite low compared with that of fresh sperm. To overcome this problem, we tried to develop new methods of sperm cryopreservation and IVF for achieving a high and stable rate of fertilization.

In our experiments, we found three kind of useful compounds for sperm cryopreservation, sperm preincubation and fertilization. Adding L-glutamine to sperm cryopreservation medium (18\% raffinose pentahydrate/3\% skim milk solution: R18S3) improved fertilization rate of frozen-thawed $\mathrm{C} 57 \mathrm{BL} / 6$ mouse sperm ${ }^{2}$. In addition, the frozen-thawed sperm which was preincubated with methyl- $\beta$-cyclodextrin dramatically activated fertility by facilitating cholestperol efflux from the cells ${ }^{3}$. Finally, reduced glutathione supported sperm penetration through zona pellucida (ZP) by changing and loosening the reticulate structure of $\mathrm{ZP}^{4)}$. Combined with these compounds for the protocol of sperm cryopreservation and IVF, we established a reliable system for producing mouse embryo via IVF using cryopreserved sperm.

In the present, our developed technology is widely used in science community. On 2011, European Mouse Mutant Archive (EMMA) which is a European community of mouse banks has been adopted our protocol of sperm cryopreservation and IVF ${ }^{5}$. The standardization of reproductive technology among mouse banks will expand and accelerate to build up a worldwide community efficiently sharing genetically engineered mice.

In conclusions, we hope our work will be contribute to facilitate the study of genetically engineered mice resulting in creating science innovations for human health.

1) Nakagata, Mammal Genome, 2000; 11, 572-576.

2) Takeo and Nakagata, Lab Animal, 2010; 44, 132-137.

3) Takeo et al. Biol Reprod, 2008; 78, 546-551.

4) Takeo and Nakagata, Biol Reprod, 2011; 85, 1066-1072.

5) European Mouse Mutant Archive, EMMA Protocols: http://www.emmanet.org/protocols/Cryopreservation-of-mouse-sperm MBCD-and-GSH-protocol_March-2012.pdf 